\title{
Relationship between uric acid and blood pressure in different age groups
}

Jae Joong Lee', Jeonghoon Ahn², Jinseub Hwang², Seong Woo Han³, Kwang No Lee', Ji Bak Kim', Sunki Lee, Jin Oh Na', Hong Euy Lim', Jin Won Kim, Seung-Woon Rha', Chang Gyu Park', Hong Seog Seo', Dong Joo Oh ${ }^{1}$ and Eung Ju Kim ${ }^{*}$

\begin{abstract}
Introduction: Serum uric acid (UA) has been known to have a positive association with blood pressure (BP). However, the relationship between serum UA and BP in different age groups is unclear.

Methods: A total of 45,098 Koreans who underwent health examinations at Korea Association of Health Promotion with no history of taking drugs related with UA and/or BP were analyzed for determining the relationship between serum UA and BP.

Results: In men $<40$, serum UA was significantly associated with systolic $(\beta=0.25, p=0.002)$ and diastolic $B P(\beta=0.41$, $p<0.001)$ after adjustment for age, diabetes, dyslipidemia, body mass index, and estimated glomerular filtration rate. Men between ages 40 and 59 showed similar results regarding diastolic BP. The association between serum UA and BP was stronger in women $<40$ ( $\beta=0.54, p<0.001$ for systolic $B P ; \beta=0.65, p<0.001$ for diastolic BP) and in between 40 and 59 ( $\beta=0.51, p<0.001$ for diastolic BP). The association was not significant in men and women $\geq 60$. The odds ratios (ORs) of hyperuricemia for hypertension were 1.25 (95\% confidence interval [Cl], 1.08 to $1.45 ; p=0.003$ ) and 1.33 ( $95 \% \mathrm{Cl}, 1.11$ to $1.60 ; p=0.002$ ) in men $<40$ and in between 40 and 59, respectively, in the multivariate analysis. The OR was $2.60(95 \% \mathrm{Cl}, 1.37$ to $4.94 ; p=0.0034)$ in women $<40$. The relationship between hyperuricemia and hypertension was not significant in other age/gender groups.
\end{abstract}

Discussion: In contrast to the elderly of 60 and over, the non-elderly showed significant associations between serum UA and BP.

Keywords: Uric acid, Hyperuricemia, Blood pressure, Hypertension

\section{Introduction}

Hypertension in adults is the most common form of cardiovascular diseases. The prevalence of hypertension grows higher with aging, resulting in an increase in morbidity and mortality through various events such as myocardial infarction, heart failure, stroke, and renal failure [1-4].

Hyperuricemia has been proposed to have an association with hypertension in various studies. Serum uric acid (UA) levels were demonstrated to be an independent predictor for developing hypertension [5-7]. Regardless of the different ethnic origins, a continuous relationship

\footnotetext{
* Correspondence: withnoel@empal.com

${ }^{1}$ Cardiovascular Center, Korea University Guro Hospital, 148 Gurodong-ro, Guro-gu, 152-703 Seoul, South Korea

Full list of author information is available at the end of the article
}

between serum UA and blood pressure (BP) was observed in African-Americans and whites [8,9] as well as in Asians [7,10] including Koreans [11-13]. For determining the causal role of serum UA in the development of hypertension, Mazzali et al. [14] demonstrated an elevation in serum UA followed by an increase in BP via a crystal-independent mechanism in rat models. Reduction of serum UA was associated with a decrease in BP through the regulation of renin-angiotensin and nitric oxide system [15].

Taking this into account, a hypothesis regarding the effect of serum UA-lowering agents which could have potential benefits in prevention and treatment of hypertension has emerged. Feig and Johnson [1] showed a high correlation between serum UA and BP in childhood primary hypertension and demonstrated promising results 
using allopurinol, a UA-lowering agent, to reduce BP in adolescents with newly developed hypertension in a pilot study [16]. Early intervention of controlling serum UA was proposed to have effect on delaying the progression of early hypertension. However, studies specifically of elderly patients have had controversial results regarding the relation between serum UA and BP $[17,18]$. Also, the use of UA-lowering agents did not have effect on controlling BP in the elderly as in the adolescents [19]. Taken together, the relationship between serum UA and BP was known to be weakened by the aging process, but there were no studies confirming in which age group the relationship would be the strongest.

Also, studies mentioned above lack control over variables which could have effect on serum UA levels such as the history of taking diuretics, antihypertensive medication or other drugs, obesity, and renal function. Moreover, some of them were performed with only a small number of subjects.

On the basis of the considerations above, we performed the present study to compare the relationship between serum UA and BP or hypertension by different age groups in a single large cohort with adjustment of all possible confounding factors. This study may identify a more specific target group in which the association between serum UA and BP or hypertension is the highest and controlling serum UA would have maximal benefit.

\section{Methods}

\section{Subjects}

A total of 91,882 subjects who underwent health examinations at Korea Association of Health Promotion (KAHP) during January, 2005 to December, 2009 were initially enrolled. Patients lacking at least 6 months of medical history in the Health Insurance Review and Assessment Service (HIRA) system were excluded. Informed consent was obtained according to the Helsinki Declaration for all of the enrolled patients in our study, and the study protocol was approved by the institutional review boards of KAHP, HIRA, and Korea University.

Subjects with a history of taking antihypertensive and/ or medications which could affect serum UA levels were also excluded. A flow diagram of the study protocol and medications for exclusion are presented in Figure 1. A total of 45,098 subjects were included in the final analysis. To evaluate the relationship between serum UA and $\mathrm{BP}$ in different ages, we divided the subjects into three groups by age; $<40,40-59$, and $\geq 60$. In each group, comparison between gender differences was done also.

\section{Baseline measurements and definitions}

Systolic and diastolic BP were measured in all of the subjects. The diagnosis of hypertension was based on a systolic BP of $140 \mathrm{mmHg}$ or higher and a diastolic BP of $90 \mathrm{mmHg}$ or higher. Height and weight were measured and body mass index (BMI) was calculated as the weight in kilograms $(\mathrm{kg})$ divided by the square of the height in meters $(\mathrm{m})$. Previous diagnoses which could affect serum UA levels, such as diabetes, chronic kidney disease, dyslipidemia, were thoroughly investigated. Fasting blood sugar levels, estimated glomerular filtration rate (eGFR), and lipid profiles including total cholesterol, triglyceride, high-density lipoprotein, and low density lipoprotein were checked for the evaluation of comorbidities. History of hospital admissions and previous medications were also taken through a cohort established between the network of KAHP and HIRA. Hyperuricemia was diagnosed in men and women each with a serum UA level over $7.0 \mathrm{mg} / \mathrm{dL}$ and $6.0 \mathrm{mg} / \mathrm{dL}$ [20].

\section{Statistical analysis}

All statistical analyses were performed using the SAS ver. 9.3 (SAS Institute Inc., Cary, NC, USA). Significance testing of the difference between means was done by an independent Student's $t$-test, and correlations were assessed by Pearson coefficients. Analysis of covariance was used

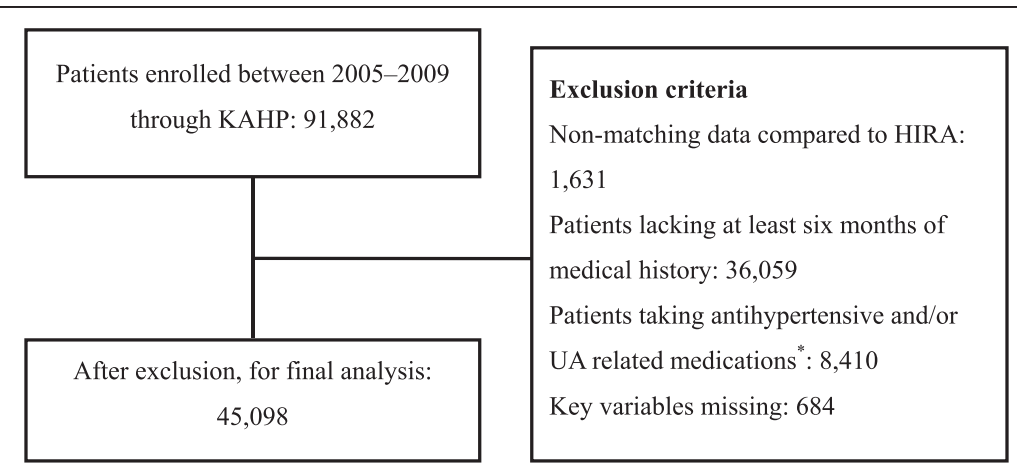

Figure 1 Flow diagram of the study protocol. *Non-antihypertensive medications which could affect serum UA levels were excluded including allopurinol, benzbromarone, colchicine, febuxostat, rasburicase, probenecid, diuretics, ethambutol, cyclosporine, diazoxide, aspirin, levodopa, and nicotinic acid. KAHP, Korea Association of Health Promotion; HIRA, Health Insurance Review and Assessment Service; UA, uric acid. 
to test for differences owing to covariates and multiple linear and logistic regression modeling to distinguish each of the contributions of age, diabetes, dyslipidemia, BMI, and eGFR to the effect of UA on BP and hyperuricemia on hypertension. All continuous variables were expressed as means \pm standard deviation. A $p$ value of $<0.05$ was considered statistically significant, but to compensate the potential of an alpha error generating from the stratified analysis regarding age and gender (a total of six groups) in the multiple linear and logistic regression model, Bonferroni correction was applied and a $p$ value of 0.05 / 6 was considered statistically significant.

\section{Results}

A total of 45,098 subjects were included in the final analysis. Baseline characteristics of the subjects divided into three groups by age are presented in Table 1 . Mean age and BMI of the total subjects were $39.0 \pm 11.6 \mathrm{~kg} / \mathrm{m}^{2}$ and $23.2 \pm 3.4 \mathrm{~kg} / \mathrm{m}^{2}$ each. Systolic BP was significantly higher in group $\geq 60$ compared to the other groups $(126.6 \pm 15.3 \mathrm{mmHg}$ vs. $114.7 \pm 12.8 \mathrm{mmHg}, 120.2 \pm$ $14.2 \mathrm{mmHg}, p<0.001)$. Comparison of diastolic BP between the three groups also showed similar results $(76.3 \pm$ $9.7 \mathrm{mmHg}$ vs. $70.8 \pm 8.9 \mathrm{mmHg}, 74.8 \pm 9.9 \mathrm{mmHg} ; p<$ $0.001)$. The prevalence of comorbidities such as hypertension, diabetes, and dyslipidemia was highest in group $\geq 60$, whereas the prevalence of hyperuricemia was highest in group $<40$. Nearly twice as many subjects were diagnosed with hypertension in hyperuricemic subjects than normouricemic subjects $(12 \%$ vs. $6 \%, p<0.001)$ (Figure 2$)$. The comparison of serum UA levels of hypertensive subjects in different age/gender groups are presented in Figure 3. Serum UA levels were significantly higher in subjects with hypertension among all the age/gender groups except $\geq 60$.

According to the multivariate linear regression analysis between serum UA and BP, in men $<40$, serum UA was significantly associated with systolic $(\beta=0.25, p=0.002)$ and diastolic BP $(\beta=0.41, p<0.001)$ after adjustment for age, diabetes, dyslipidemia, BMI, and eGFR. However, men between the age 40 and 59 showed similar results regarding only diastolic $\mathrm{BP}(\beta=0.43, p<0.001)$. The association between serum UA and BP was stronger in women $<40(\beta=0.54, p<0.001$ for systolic BP; $\beta=0.65$, $p<0.001$ for diastolic BP) and in between 40 and 59 regarding diastolic $\mathrm{BP}(\beta=0.51, p<0.001)$. The association was not significant in men and women $\geq 60$ (Table 2 ).

After performing multiple logistic regression analysis, the odds ratios (ORs) of hyperuricemia for hypertension were 1.25 (95\% confidence interval $[\mathrm{CI}], 1.08$ to $1.45 ; p=$ 0.003 ) and 1.33 (95\% CI, 1.11 to $1.60 ; p=0.002$ ) each in men $<40$ and in 40 to 59 , respectively. In women $<40$, the

Table 1 Baseline characteristics of each group by age

\begin{tabular}{|c|c|c|c|c|c|}
\hline Variable & $\begin{array}{l}\text { Total } \\
(n=45,098)\end{array}$ & $\begin{array}{l}<40 \text { years } \\
(n=28,864)\end{array}$ & $\begin{array}{l}40-59 \text { years } \\
(n=13,118)\end{array}$ & $\begin{array}{l}\geq 60 \text { years } \\
(n=3,116)\end{array}$ & $p$ value \\
\hline Male & $24,031(53.3)$ & $15,212(52.7)$ & $7,089(54.0)$ & $1,730(55.5)$ & $<0.001$ \\
\hline Age (years) & $39.0 \pm 11.6$ & $31.7 \pm 4.9$ & $48.7 \pm 5.5$ & $65.0 \pm 4.2$ & $<0.001$ \\
\hline Height $(\mathrm{cm})$ & $165.6 \pm 8.7$ & $167.2 \pm 8.5$ & $163.5 \pm 8.3$ & $160.0 \pm 8.6$ & $<0.001$ \\
\hline Weight (kg) & $63.9 \pm 12.6$ & $64.3 \pm 13.7$ & $63.7 \pm 10.2$ & $60.5 \pm 9.5$ & $<0.001$ \\
\hline Body mass index $\left(\mathrm{kg} / \mathrm{m}^{2}\right)$ & $23.2 \pm 3.4$ & $22.9 \pm 3.7$ & $23.7 \pm 2.8$ & $23.6 \pm 2.9$ & $<0.001$ \\
\hline Systolic blood pressure (mmHg) & $117.1 \pm 13.9$ & $114.7 \pm 12.8$ & $120.2 \pm 14.2$ & $126.6 \pm 15.3$ & $<0.001$ \\
\hline Diastolic blood pressure (mmHg) & $72.3 \pm 9.5$ & $70.8 \pm 8.9$ & $74.8 \pm 9.9$ & $76.3 \pm 9.7$ & $<0.001$ \\
\hline Uric acid (mg/dL) & $5.2 \pm 1.5$ & $5.3 \pm 1.5$ & $5.1 \pm 1.4^{*}$ & $5.1 \pm 1.3^{*}$ & $<0.001$ \\
\hline Fasting blood sugar (mg/dL) & $91.3 \pm 17.7$ & $89.3 \pm 14.6$ & $94.4 \pm 20.1$ & $96.6 \pm 21.4$ & $<0.001$ \\
\hline Estimated glomerular filtration rate $\left(\mathrm{mL} / \mathrm{min} / 1.73 \mathrm{~m}^{2}\right)$ & $88.0 \pm 27.1$ & $91.2 \pm 28.0$ & $83.1 \pm 24.6$ & $78.4 \pm 23.4$ & $<0.001$ \\
\hline Total cholesterol (mg/dL) & $180.2 \pm 33.8$ & $174.2 \pm 31.3$ & $189.9 \pm 33.7$ & $194.4 \pm 34.4$ & $<0.001$ \\
\hline High-density lipoprotein (mg/dL) & $52.0 \pm 10.2$ & $52.1 \pm 10.0^{*}$ & $51.8 \pm 10.4^{\dagger}$ & $52.0 \pm 10.4^{*, \dagger}$ & 0.046 \\
\hline Triglyceride (mg/dL) & $105.6 \pm 82.5$ & $100.1 \pm 62.2$ & $115.4 \pm 67.0$ & $115.3 \pm 60.0$ & $<0.001$ \\
\hline Low density lipoprotein (mg/dL) & $107.1 \pm 29.3$ & $102.3 \pm 27.2$ & $115.0 \pm 30.7$ & $117.3 \pm 31.2$ & $<0.001$ \\
\hline Hypertension & $3,122(6.9)$ & $1,144(3.9)$ & $1,377(10.5)$ & $601(19.3)$ & $<0.001$ \\
\hline Diabetes mellitus & $1,224(2.7)$ & $344(1.2)$ & $625(4.8)$ & $255(8.2)$ & $<0.001$ \\
\hline Dyslipidemia & $10,073(22.3)$ & $5,330(18.5)$ & $3,752(28.6)$ & 991 (31.8) & $<0.001$ \\
\hline Hyperuricemia & $5,700(12.6)$ & $4,104(14.2)$ & $1,296(9.9)$ & $300(9.6)$ & $<0.001$ \\
\hline
\end{tabular}

Values are presented as number (\%) or mean \pm standard deviation.

All $p$ values in the post hoc analysis comparing each group in all the variables were under 0.05 except the groups indicated as same letters like ${ }^{*}$ or $t$. 


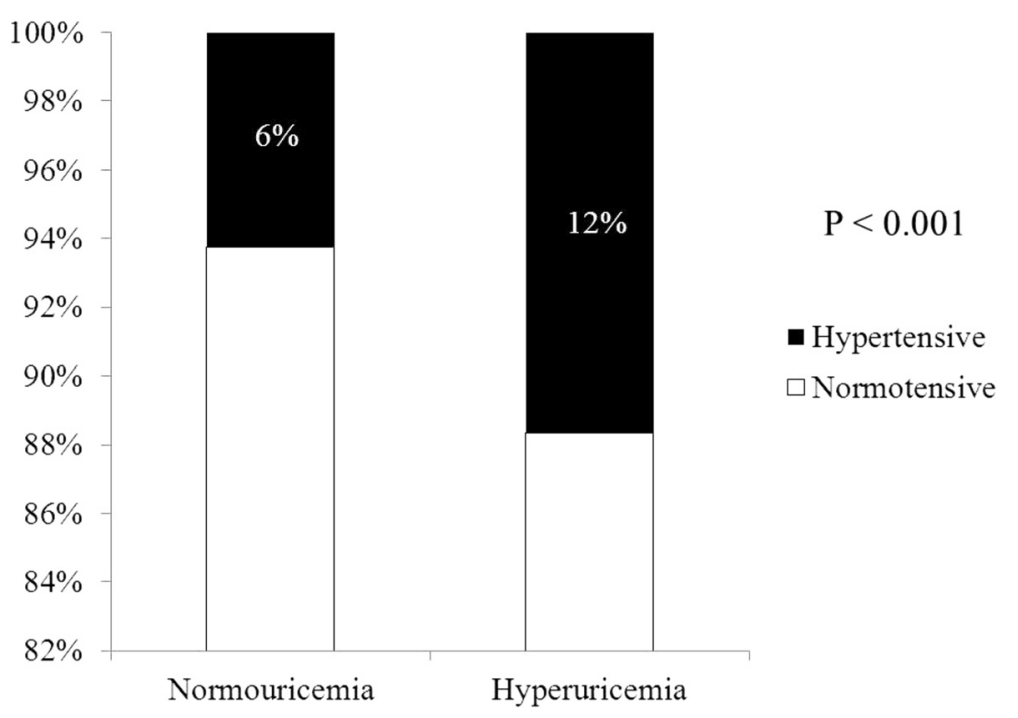

Figure 2 Prevalence of hypertension in hyperuricemia. A significant difference between normouricemic and hyperuricemic patients was seen among the total subjects who were enrolled $(p<0.05)$.

OR was 2.60 ( $95 \%$ CI, 1.37 to $4.94 ; p=0.003$ ). The relationship between hyperuricemia and hypertension was not significant in other age/gender groups (Table 3).

\section{Discussion}

The major findings in the present study enable us to identify the importance of age in the relation between serum UA and BP. Although serum UA and BP showed a significant relation in the overall population, when we evaluated it according to different age groups $(<40,40-$ $59, \geq 60$ ), it was only significant in the non-elderly population under age 60 in both genders. There have been several studies suggesting that the strength of the relationship between serum UA and BP is more dominant in the younger age groups and decreases during the aging process as the duration of hypertension gets longer
[1,6,12,21-23]. However, none of the previous studies have tried to investigate the effect of different age groups on the relation as a primary goal. To our knowledge, this is the first study to confirm the effect of different age groups on the relation between serum UA and BP in a single large cohort.

The prognostic significance of serum UA in different disease entities such as diabetes, chronic kidney disease, and cardiovascular diseases have been recognized in several previous studies $[2,5,6,9,24,25]$. Understanding the relationship between each diseases and serum UA has been important due to the potential benefit which could be achieved by applying it to new treatment strategies. Feig et al. [16] mentioned that early hypertension developed in children and adolescents with hyperuricemia could be reversed with urate reduction. Considering the

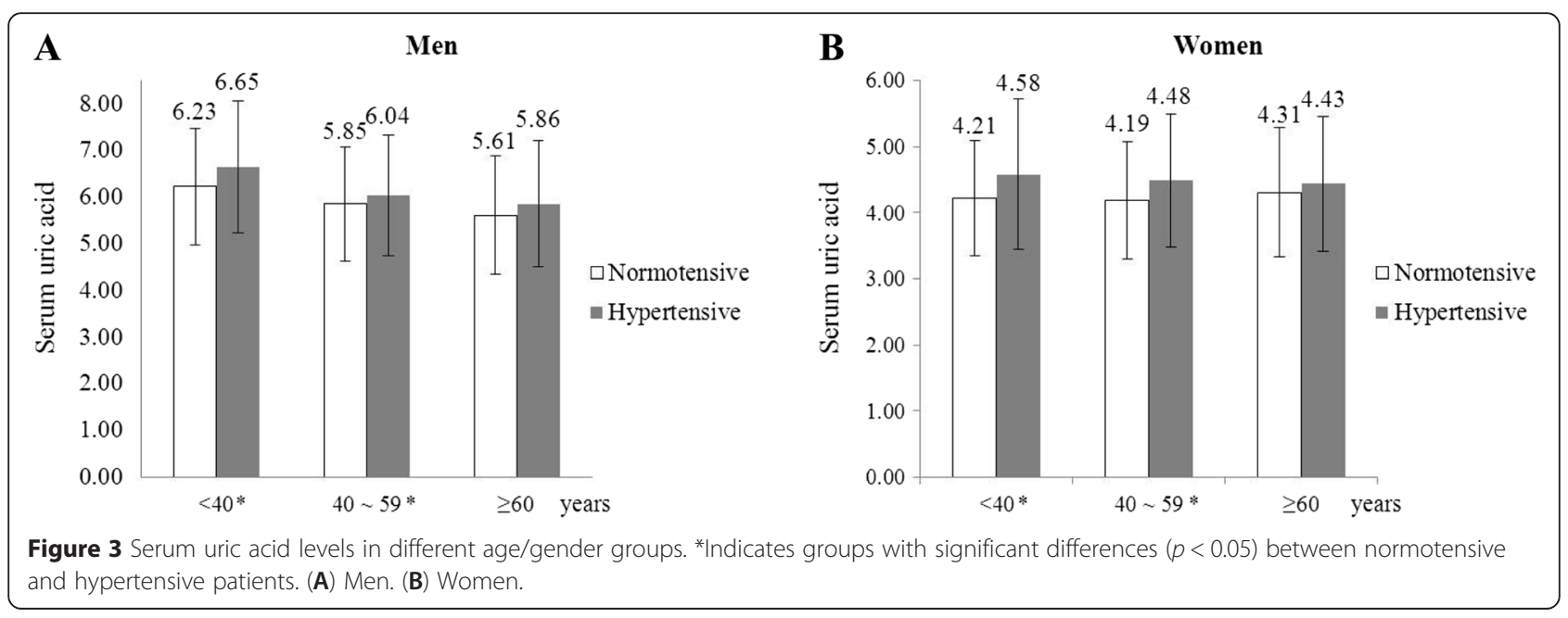


Table 2 Multivariate linear regression analysis between blood pressure and uric acid

\begin{tabular}{|c|c|c|c|c|c|c|c|c|c|c|c|c|}
\hline \multirow[t]{3}{*}{ Groups } & \multicolumn{6}{|l|}{ Men } & \multicolumn{6}{|c|}{ Women } \\
\hline & \multicolumn{3}{|l|}{ SBP } & \multicolumn{3}{|l|}{ DBP } & \multicolumn{3}{|l|}{ SBP } & \multicolumn{3}{|l|}{ DBP } \\
\hline & $\beta$ & $R^{2}$ & $p$ value & $\beta$ & $R^{2}$ & $p$ value & $\beta$ & $R^{2}$ & $p$ value & $\beta$ & $R^{2}$ & $p$ value \\
\hline Total & 0.33 & 0.10 & $<0.001$ & 0.39 & 0.10 & $<0.001$ & 0.61 & 0.19 & $<0.001$ & 0.60 & 0.14 & $<0.001$ \\
\hline$<40$ years & 0.25 & 0.09 & 0.002 & 0.40 & 0.09 & $<0.001$ & 0.54 & 0.05 & $<0.001$ & 0.65 & 0.04 & $<0.001$ \\
\hline 40-59 years & 0.29 & 0.06 & 0.03 & 0.43 & 0.05 & $<0.001$ & 0.44 & 0.12 & 0.04 & 0.51 & 0.09 & $<0.001$ \\
\hline$\geq 60$ years & 0.57 & 0.05 & 0.05 & 0.34 & 0.03 & 0.08 & -0.67 & 0.07 & 0.12 & -0.26 & 0.04 & 0.35 \\
\hline
\end{tabular}

Adjustment was done with age, diabetes, dyslipidemia, body mass index, and estimated glomerular filtration rate. SBP systolic blood pressure, DBP diastolic blood pressure.

practical implications in every day clinical practice, our results suggest the same could be applied beyond the adolescent to the non-elderly adults under age 60 with early hypertension and hyperuricemia.

There are several potential explanations for the attenuation of the relationship in the elderly. Previous studies implicating similar results have mentioned that the effect of a single estimate of serum UA in the young may decrease over time, and the higher background rate of hypertension incidence by other causes with increasing age may contribute to the reduction of the strength of the relationship between serum UA and BP [21]. Although most of the studies controlled possible confounding variables such as age, BMI, and serum cholesterol levels, many of them did not adjust for diabetes [6,25], antihypertensive therapy [24-26], or diuretic use [25-27] which led to controversial results regarding the relationship between serum UA and BP, especially in the elderly. However, our study initially was designed for complete control over the variables mentioned above and excluded patients taking medications possibly affecting serum UA levels and BP. Variables such as age, BMI, diabetes, dyslipidemia, and renal function, represented by eGFR, were all taken into account for adjustment in the final analysis. Even after the adjustments, the current study confirmed a significant correlation with serum UA and BP in the non-elderly patients under age 60. Hyperuricemia increased the risk of hypertension in both genders, but was only significant in men $<60$ and women $<40$ in the multiple logistic regression

Table 3 Multivariate logistic regression analysis between hyperuricemia and hypertension in different age/gender groups

\begin{tabular}{lllllllll}
\hline Groups & \multicolumn{3}{l}{ Men } & & \multicolumn{5}{l}{ Women } \\
\cline { 2 - 3 } \cline { 7 - 8 } & OR & $\mathbf{C l}$ & $\boldsymbol{p}$ value & & OR & $\mathbf{C l}$ & $\boldsymbol{p}$ value \\
\hline Total & 1.29 & $1.16-1.44$ & $<0.001$ & & 1.35 & $0.99-1.84$ & 0.06 \\
$<40$ years & 1.25 & $1.08-1.45$ & 0.003 & & 2.60 & $1.37-4.94$ & 0.003 \\
40-59 years & 1.33 & $1.11-1.60$ & 0.002 & & 1.26 & $0.81-1.95$ & 0.31 \\
$\geq 60$ years & 1.29 & $0.93-1.79$ & 0.13 & & 1.17 & $0.65-2.09$ & 0.61 \\
\hline
\end{tabular}

Adjustment was done with age, diabetes, dyslipidemia, body mass index, and estimated glomerular filtration rate.

$O R$ odds ratio, $\mathrm{Cl}$ confidence interval. analysis. Particularly in women younger than 40 with hyperuricemia, our study demonstrated a 2.6-fold increase in the risk of hypertension. Stronger association of serum UA with BP and hypertension in women was seen, which is in line with previous studies [6,23,28,29]. Awareness and early interventional strategies in controlling hyperuricemia associated with BP reduction in this specific group may obtain maximal benefit.

Studies using animal models and cell cultures have revealed mechanisms by which UA could cause hypertension. In short, hypertension was developed by UAmediated renal vasoconstriction resulting from a reduction in endothelial levels of nitric oxide, with activation of renin-angiotensin system [30,31]. Microvascular renal disease independently was caused by UA over time, inducing the development of hypertension [30].

Recent therapeutic trials with UA-lowering agents have demonstrated promising results for reducing BP. In one small study, the use of allopurinol, a xanthine oxidase $(\mathrm{XO})$ inhibitor, resulted in reduction of $\mathrm{BP}$ in adolescents with newly diagnosed hypertension with hyperuricemia [16]. In another small study, UA-lowering therapy with either allopurinol or probenecid, a uricosuric agent, significantly reduced BP in prehypertensive, obese, adolescents with hyperuricemia irrespective of the UA-lowering mechanisms [32]. Allopurinol also showed significant additional BP reduction when it was combined with enalapril compared to enalapril alone in hyperuricemic hypertensive adolescents [33]. There was a report that a 6-month treatment of febuxostat, a more recently developed selective XO inhibitor, showed a significant $\mathrm{BP}$ reduction even in the elderly patients (mean age, $67 \pm 10.3$ years) with serum $U A \geq 8 \mathrm{mg} / \mathrm{dL}$ in contrast to allopurinol [34]. Febuxostat had a stronger UAlowering effect and a renoprotective effect and was also superior to allopurinol at inhibiting oxidative stress atherogenesis, hypertension, and vascular endothelial damage. However, the study was performed with an intermediate size specific group who underwent cardiac surgery, and $90 \%$ of the patients were already being treated with antihypertensive therapy. Therefore, the effect of febuxostat on BP reduction may not be applicable 
to the general hyperuricemic population including the elderly, yet.

Our study has several limitations. First, its crosssectional design could not reveal the causal role of serum UA in the development of hypertension. Increased UA levels at baseline are suggested to result in vascular dysfunction but after chronic exposure to high UA levels, the corresponding alterations in the vasculature diminish over time [35]. There may be an underlying genetic difference between genders but the results of this study demonstrating a stronger association between UA and hypertension in women than in men could have occurred due to the difference in the duration of exposure to elevated UA levels [36]. Second, in contrast with previous studies [37], the prevalence of hyperuricemia was highest in group $<40$. In previous studies, the increasing prevalence of hyperuricemia in the elderly was because of higher incidence of taking antihypertensive and/or diuretic agents [7,17]. The exclusion of these patients may have led to the results above. Third, the statistical insignificance in the elderly may have been due to the relatively smaller sample size compared to the other groups. Fourth, with the exclusion of subjects on antihypertensive medications, this may have generated a bias in which our study results only regard subjects with mild hypertension. However, our distinct study design compared to previous observational studies [24-27] in which we completely controlled medications affecting serum UA levels may provide a more accurate interpretation in the relationship between serum UA and BP. Finally, we cannot absolutely exclude the possibility that the study subjects were taking medications which could affect serum UA levels or BP from family or friends in secrecy because we only evaluated the medication history based on the legal prescriptions recorded by HIRA.

\section{Conclusions}

This single large cohort study confirmed that the relationship between serum UA and BP, hyperuricemia and hypertension is quite different in each age group. In contrast to the elderly of 60 and over, the non-elderly showed a significant association between serum UA and $\mathrm{BP}$ in both genders. Hyperuricemia increased the relative risk of hypertension by approximately $30 \%$ in men under 60 and by 2.6 fold in women under 40 . We believe the present study suggests specific age/gender groups which would obtain maximal benefit from the treatment of hyperuricemia for the prevention and treatment of hypertension.

\section{Competing interests}

The authors declare that they have no competing interests.

\section{Acknowledgements}

We thank Yea Ji Jung and Min-Jeong Kim at the National Evidence-based Healthcare Collaborating Agency (NECA) for their astonishing work and Korea Association of Health Promotion (KAHP) for their full support.

\section{Source of funding}

This research was supported in part by a grant through the National Evidence-based Healthcare Collaborating Agency (NA11-005) and the Korean Society of Hypertension.

\section{Author details}

${ }^{1}$ Cardiovascular Center, Korea University Guro Hospital, 148 Gurodong-ro, Guro-gu, 152-703 Seoul, South Korea. ${ }^{2}$ National Evidence-based Healthcare Collaborating Agency, Seoul, South Korea. ${ }^{3}$ Department of Cardiology, Dongtan Sacred Heart Hospital, Hallym University Medical Center, Hwaseong, South Korea.

Received: 22 April 2015 Accepted: 22 April 2015

Published online: 15 July 2015

\section{References}

1. Feig DI, Johnson RJ. Hyperuricemia in childhood primary hypertension. Hypertension. 2003;42:247-52.

2. Coresh J, Wei GL, McQuillan G, Brancati FL, Levey AS, Jones C, et al. Prevalence of high blood pressure and elevated serum creatinine level in the United States: findings from the third National Health and Nutrition Examination Survey (1988-1994). Arch Intern Med. 2001;161:1207-16.

3. Park JK. Epidemiology of hypertension. J Korean Soc Hypertens. 1995;1:6-17.

4. Kim JS, Lee HC, Yoo WS, Yoo UH. Mean blood pressure, prevalence and epidemiologic characteristics of hypertension among representative Korean adult population. J Korean Soc Hypertens. 1998:4:89-98.

5. Jossa F, Farinaro E, Panico S, Krogh V, Celentano E, Galasso R, et al. Serum uric acid and hypertension: the Olivetti heart study. J Hum Hypertens. 1994;8:677-81

6. Brand FN, McGee DL, Kannel WB, Stokes 3rd J, Castelli WP. Hyperuricemia as a risk factor of coronary heart disease: the Framingham study. Am J Epidemiol. 1985;121:11-8.

7. Kansui Y, Ohtsubo T, Goto K, Sakata S, Ichishima K, Fukuhara M, et al. Association of serum uric acid with blood pressure in Japanese men. Crosssectional study in work-site group. Circ J. 2011;75:2827-32.

8. Longo-Mbenza B, Luila EL, Mbete P, Vita EK. Is hyperuricemia a risk factor of stroke and coronary heart disease among Africans? Int J Cardiol. 1999;71:17-22.

9. Klein R, Klein BE, Cornoni JC, Maready J, Cassel JC, Tyroler HA. Serum uric acid: its relationship to coronary heart disease risk factors and cardiovascular disease, Evans County, Georgia. Arch Intern Med. 1973;132:401-10.

10. Kuwabara M, Niwa K, Nishi Y, Mizuno A, Asano T, Masuda K, et al. Relationship between serum uric acid levels and hypertension among Japanese individuals not treated for hyperuricemia and hypertension. Hypertens Res. 2014;37:785-9.

11. Kim YH, Suh YD, Son SP, Shim YW, Shin YW, Shin YK, et al. Observation of the serum uric acid in essential hypertension. Korean J Med. 1985;28:56-63.

12. Chin HJ, Na KY, Kim Y, Chae DW, Kim S. The impact of uric acid and metabolic syndrome on the incidence of hypertension in a Korean population. Korean J Med. 2007;73:58-66.

13. Yoo TW, Sung KC, Kim YC, Hwang ST, Oh SY, Shin HS, et al. The relationship of the hypertension, insulin resistance, and metabolic syndrome in the serum uric acid level. Korean Circ J. 2004:34:874-82.

14. Mazzali M, Hughes J, Kim YG, Jefferson JA, Kang DH, Gordon KL, et al. Elevated uric acid increases blood pressure in the rat by a novel crystalindependent mechanism. Hypertension. 2001;38:1101-6.

15. Mazzali M, Kanellis J, Han L, Feng L, Xia YY, Chen Q, et al. Hyperuricemia induces a primary renal arteriolopathy in rats by a blood pressureindependent mechanism. Am J Physiol Renal Physiol. 2002;282:F991-7.

16. Feig DI, Soletsky B, Johnson RJ. Effect of allopurinol on blood pressure of adolescents with newly diagnosed essential hypertension: a randomized trial. JAMA. 2008;300:924-32.

17. Culleton BF, Larson MG, Kannel WB, Levy D. Serum uric acid and risk for cardiovascular disease and death: the Framingham heart study. Ann Intern Med. 1999;131:7-13.

18. Staessen J. The determinants and prognostic significance of serum uric acid in elderly patients of the European working party on high blood pressure in the elderly trial. Am J Med. 1991;90:50S-4.

19. Siu YP, Leung KT, Tong MK, Kwan TH. Use of allopurinol in slowing the progression of renal disease through its ability to lower serum uric acid level. Am J Kidney Dis. 2006;47:51-9. 
20. Lin KC, Lin HY, Chou P. Community based epidemiological study on hyperuricemia and gout in Kin-Hu, Kinmen. J Rheol. 2000;27:1045-50.

21. Sundstrom J, Sullivan L, D'Agostino RB, Levy D, Kannel WB, Vasan RS Relations of serum uric acid to longitudinal blood pressure tracking and hypertension incidence. Hypertension. 2005:45:28-33.

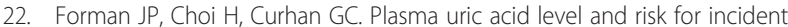
hypertension among men. J Am Soc Nephrol. 2007;18:287-92.

23. Grayson PC, Kim SY, LaValley M, Choi HK. Hyperuricemia and incident hypertension: a systematic review and meta-analysis. Arthritis Care Res (Hoboken). 2011;63:102-10.

24. Bengtsson C, Lapidus L, Stendahl C, Waldenstrom J. Hyperuricaemia and risk of cardiovascular disease and overall death: a 12-year follow-up of participants in the population study of women in Gothenburg, Sweden. Acta Med Scand. 1988;224:549-55

25. Levine W, Dyer AR, Shekelle RB, Schoenberger JA, Stamler J. Serum uric acid and 11.5-year mortality of middle-aged women: findings of the Chicago Heart Association detection project in industry. J Clin Epidemiol. 1989:42:257-67.

26. Yano K, Reed DM, McGee DL. Ten-year incidence of coronary heart disease in the Honolulu heart program: relationship to biologic and lifestyle characteristics. Am J Epidemiol. 1984;119:653-66.

27. Reunanen A, Takkunen $\mathrm{H}$, Knekt $\mathrm{P}$, Aromaa A. Hyperuricemia as a risk factor for cardiovascular mortality. Acta Med Scand Suppl. 1982;668:49-59.

28. Nagahama K, Inoue T, Iseki $K$, Touma T, Kinjo K, Ohya $Y$, et al. Hyperuricemia as a predictor of hypertension in a screened cohort in Okinawa, Japan. Hypertens Res. 2004;27:835-41.

29. Alper Jr AB, Chen W, Yau L, Srinivasan SR, Berenson GS, Hamm LL. Childhood uric acid predicts adult blood pressure: the Bogalusa heart study. Hypertension. 2005:45:34-8.

30. Feig DI, Kang DH, Johnson RJ. Uric acid and cardiovascular risk. N Engl J Med. 2008;359:1811-21.

31. Johnson RJ, Sanchez-Lozada LG, Mazzali M, Feig DI, Kanbay M, Sautin YY What are the key arguments against uric acid as a true risk factor for hypertension? Hypertension. 2013;61:948-51

32. Soletsky B, Feig DI. Uric acid reduction rectifies prehypertension in obese adolescents. Hypertension. 2012;60:1148-56.

33. Assadi F. Allopurinol enhances the blood pressure lowering effect of enalapril in children with hyperuricemic essential hypertension. J Nephrol. 2014;27:51-6.

34. Shibagaki Y, Ohno I, Hosoya T, Kimura K. Safety, efficacy and renal effect of febuxostat in patients with moderate-to-severe kidney dysfunction. Hypertens Res. 2014;37:919-25.

35. Feig DI, Madero M, Jalal DI, Sanchez-Lozada LG, Johnson RJ. Uric acid and the origins of hypertension. J Pediatr. 2013;162:896-902

36. Samimi A, Ramesh S, Turin TC, MacRae JM, Sarna MA, Reimer RA, et al Serum uric acid level, blood pressure, and vascular angiotensin II responsiveness in healthy men and women. Physiol Rep. 2014;2:e12235.

37. Wallace KL, Riedel AA, Joseph-Ridge N, Wortmann R. Increasing prevalence of gout and hyperuricemia over 10 years among older adults in a managed care population. J Rheol. 2004;31:1582-7.

\section{Submit your next manuscript to BioMed Central and take full advantage of:}

- Convenient online submission

- Thorough peer review

- No space constraints or color figure charges

- Immediate publication on acceptance

- Inclusion in PubMed, CAS, Scopus and Google Scholar

- Research which is freely available for redistribution 